\title{
SOBREVIDA GLOBAL E OUTROS DESFECHOS CLÍNICOS EM CÂNCER DE MAMA: SITUAÇÃO ATUAL E CONTROVÉRSIAS
}

Karime K. Machado ${ }^{1}$, Artur Katz ${ }^{2}$, Marc Buyse ${ }^{3}$, Everardo Delforge Saad ${ }^{4 *}$

Trabalho realizado na Dendrix, São Paulo, SP

\section{INTRODUÇão}

Em virtude dos avanços recentes no tratamento sistêmico do câncer de mama, um número progressivamente maior de drogas e de combinações e sequências terapêuticas estão sendo avaliadas e tornando-se disponíveis para uso clínico nas diversas linhas de tratamento na doença avançada. ${ }^{1-4}$ Apesar da crescente disponibilidade de opções para pacientes e médicos, a escolha dos desfechos clínicos utilizados nos estudos vem ficando mais complexa; essa escolha é crítica para o desenvolvimento de novos fármacos e para progressos futuros. ${ }^{5}$ Porém, existe grande controvérsia atual a respeito de quais desfechos usados em estudos clínicos mais bem representam o benefício associado à intervenção terapêutica de interesse. Recentemente, nosso grupo realizou alguns estudos a respeito desse tema, e um dos trabalhos, publicado no periódico Journal of Clinical Oncoloy, ${ }^{6}$ motivou a preparação do presente artigo, no qual apresentamos os principais achados de nossa linha de pesquisa e algumas opiniões sobre esse assunto.

\section{Desfechos temporais EM ONCOLOGIA}

Desfechos clínicos como sobrevida global, sobrevida livre de progressão e tempo para progressão são os mais usados em Oncologia, ao menos nas fases avançadas de desenvolvimento clínico de uma nova droga ou combinação. Esses desfechos fornecem informações não só a respeito da ocorrência ou não de eventos como morte e progressão de doença, mas também do momento em que esses eventos ocorrem.

O método de Kaplan-Meier ${ }^{7}$ é o mais utilizado para a análise desses desfechos. Nesse método não-paramétrico, os eventos de interesse e as razões para censura dos pacientes na análise são primariamente definidos. Na censura, excluem-se do denominador os pacientes que não apresentaram o evento de interesse por ocasião da análise, no último momento em que se sabia que estes pacientes estavam sob risco para a sua ocorrência, já que informações adicionais sobre esses indivíduos não estariam disponíveis a partir do momento da censura. Uma vez definidos os eventos de interesse e as razões para censura, é possível construir curvas de Kaplan-Meier que podem ser úteis para descrever a taxa de ocorrência dos desfechos ao longo do tempo, bem para comparar essas taxas em diferentes grupos de pacientes. A Tabela 1 mostra as definições de eventos e as razões para censura nas análises mais usadas em oncologia.

Tendo em vista que os principais objetivos do tratamento em câncer de mama avançado são o aumento da sobrevida e a melhoria da qualidade de vida $^{8}$ a sobrevida global é historicamente considerada o desfecho terapêutico de maior importância. ${ }^{9}$ Neste caso, o evento de interesse é a morte por quaisquer causas, e as pacientes são censurados apenas quando ocorre perda de seguimento ou quando elas ainda estão vivas por ocasião da análise.

\begin{tabular}{|c|c|c|}
\hline Desfecho clínico & Evento(s) de interesse & Razões para a censura \\
\hline Sobrevida global & Morte por quaisquer causas & Término do seguimento (paciente vivo) ou perda de seguimento \\
\hline Sobrevida livre de progressão & $\begin{array}{l}\text { Progressão de doença ou morte por quaisquer } \\
\text { causas }\end{array}$ & $\begin{array}{l}\text { Término do seguimento (paciente vivo e sem progressão de doença) } \\
\text { ou perda de seguimento }\end{array}$ \\
\hline Tempo para progressão & $\begin{array}{l}\text { Progressão da doença (para alguns autores, } \\
\text { morte por câncer ou morte por quaisquer causas } \\
\text { são considerados eventos) }\end{array}$ & $\begin{array}{l}\text { Término do seguimento (paciente vivo e sem progressão de } \\
\text { doença), morte sem documentação de progressão prévia, ou perda } \\
\text { de seguimento }\end{array}$ \\
\hline
\end{tabular}

1 - Médica Oncologista - Médica Residente do Instituto do Câncer do Estado de São Paulo, São Paulo, SP

2- Médico Oncologista do Centro de Oncologia, Hospital Sírio-Libanês, São Paulo, São Paulo, SP

3- ScD Estatístico e Diretor do International Drug Development Institute, Louvain-la-Neuve, Bélgica

4- Médico - Diretor Científico da Dendrix, São Paulo, SP 


\section{Controvérsia A RESPEITO dA SOBREVIDA GLOBAL}

Recentemente, o uso da sobrevida global como desfecho primário em oncologia tem sido questionado, uma vez que apresenta desvantagens, tais como o longo tempo de seguimento necessário para sua avaliação e o fato de a sobrevida ser influenciada pela ocorrência de morte por outras causas que não o câncer ou o tratamento. ${ }^{10}$ Mais importante do que isso, a sobrevida é influenciada pelo cruzamento de pacientes entre os diferentes tratamentos do estudo e pelo efeito não quantificado de tratamentos potencialmente ativos usados em linhas posteriores ao término do estudo. ${ }^{11}$ Isto é observado especialmente no tratamento do câncer colorretal ${ }^{12}$ e do câncer de mama, doenças em que diversas linhas de tratamento eficazes encontram-se disponíveis.

A sobrevida livre de progressão consiste no tempo decorrido entre o início do tratamento e a progressão de doença ou morte por quaisquer causas ${ }^{13}$. A censura também é feita quando ocorre perda de seguimento ou quando os pacientes encontram-se vivos e sem progressão por ocasião da análise. Um desfecho análogo, o tempo para progressão de doença, consiste no tempo transcorrido entre o início do tratamento e a progressão de doença, com censura se houver morte por quaisquer causas (Tabela 1). Em teoria, o evento de interesse é exclusivamente a progressão de doença ${ }^{1}$; entretanto, há estudos em que se considera como evento a morte pelo câncer. ${ }^{14}$ Para fins regulatórios, tende-se a preferir a sobrevida livre de doença como desfecho mais importante que o tempo para progressão, pois a primeira pode detectar a ocorrência de toxicidade fatal pelo tratamento. ${ }^{1}$

Na última década, diversos investigadores utilizaram a sobrevida livre de progressão e o tempo para progressão de forma intercambiável, ${ }^{14}$ ao considerarem morte por quaisquer causas como evento em ambos os casos. O que se observa nos estudos clínicos é que a maioria dos pacientes com um evento na análise da sobrevida livre de progressão apresenta progressão de doença antes da morte. ${ }^{14}$ Portanto, na prática, é possível que a sobrevida livre de progressão e o tempo para progressão sejam idênticos, mas ainda assim há necessidade de uniformização dos termos utilizados e de especificação das causas de censura nos manuscritos. ${ }^{1}$ Há ainda outros desfechos clínicos tempo-dependentes, mais utilizados como desfechos secundários.

Segundo análise da literatura recente, ${ }^{15}$ os estudos clínicos em câncer de mama avançado têm priorizado o uso da sobrevida livre de progressão e o tempo para progressão como desfechos primários, em detrimento à sobrevida global. Entretanto, esses desfechos nem sempre se correlacionam positivamente com a sobrevida, e há discordância na literatura quanto a seu valor como desfechos sucedâneos, ${ }^{10,16-18}$ os quais necessitam de validação para cada linha de tratamento em cada tipo de neoplasia.

\section{Uso de sobrevida global na literatura recente}

O estudo em questão teve como objetivo avaliar os principais desfechos utilizados em estudos randomizados controlados de tratamento do câncer de mama avançado nos últimos 10 anos. ${ }^{6}$ Para tanto, realizamos um levantamento bibliográfico, usando PubMed, de estudos publicados nos 11 principais periódicos que divulgam estudos clínicos sobre câncer de mama no período de $1^{\circ}$ de Janeiro de 1998 a 31 de Dezembro de 2007.
Encontramos 76 artigos que preenchiam os critérios de busca. Os estudos de fase III em questão incluíram o total de 29.442 pacientes, com mediana de 327 pacientes por estudo. Cerca de $60 \%$ dos estudos avaliaram tratamentos de primeira linha, e em $49 \%$ deles foram comparados diferentes regimes de quimioterapia, excluindo-se tratamentos hormonais e com drogas de alvo molecular. Os desfechos primários utilizados foram sobrevida livre de progressão, tempo para progressão ou tempo para falha do tratamento, em 39 estudos (51\%), taxa de resposta objetiva, em 31 casos (40\%), e sobrevida global em apenas quatro estudos (5\%). Apesar de ter sido pouco usada como desfecho primário, em $84,2 \%$ da amostra a sobrevida global estava entre os cinco primeiros desfechos clínicos secundários avaliados.

A média de sobrevida mediana nos estudos avaliados foi de 20,5 meses, sendo ainda maior nos estudos que continham tratamentos hormonais (27,4 meses). Em quinze estudos $(19,7 \%)$, observou-se diferença estatisticamente significativa em sobrevida quando dois ou mais grupos de tratamentos foram comparados. Essa diferença variou de 1,8 a 8 meses, com mediana de 4,7 meses.

Dados combinados de sobrevida livre de progressão e de sobrevida global estavam disponíveis em 64 estudos, dos quais 31 (48\%) mostraram benefício em sobrevida livre de progressão, tendo-se documentado benefício em ambos os desfechos em 13 estudos (20\%). Houve, portanto, benefício em sobrevida global em $41 \%$ desses estudos em que houve benefício em sobrevida livre de progressão. Verificamos também que a mediana de sobrevida após progressão da doença, calculada como a mediana de sobrevida global menos a mediana de sobrevida livre de progressão para cada braço de todos os estudos, foi de 13,6 meses, correspondendo a dois terços do tempo de sobrevida das pacientes após início do tratamento. A sobrevida após progressão da doença foi maior no subgrupo de pacientes que receberam hormonioterapia (20,5 meses), o que pode ser justificado pela história natural mais indolente da doença positiva para receptores hormonais. Curiosamente, porém, houve relativamente pouca variabilidade da sobrevida após progressão da doença, quando esse parâmetro foi considerado de maneira percentual. Dessa forma, constatamos que a sobrevida após progressão da doença representa em média dois terços do tempo de sobrevida das pacientes incluídas em estudos clínicos, independentemente da linha ou tipo de tratamento.

Apesar da susceptibilidade ao viés de publicação, o estudo em questão mostra que a sobrevida global é um desfecho primário pouco utilizado, embora haja benefício estatisticamente significativo em cerca de $20 \%$ dos estudos, o que não é pouco nesse contexto. Além disso, o benefício em sobrevida livre de progressão costuma preceder o benefício em sobrevida, indicando seu possível papel como desfecho sucedâneo. Em contrapartida, a taxa de resposta é desfecho primário em uma grande proporção dos estudos, ainda que esse não possa ser considerado um desfecho sucedâneo de sobrevida em câncer de mama. ${ }^{17}$

Juntamente com outros estudos semelhantes publicados recentemente, esses achados podem contribuir para a prática médica, na medida em que auxiliam o oncologista clínico a determinar a relevância de desfechos usados nos estudos clínicos e confrontar os resultados desses estudos com os objetivos 
terapêuticos dos tratamentos oferecidos a seus pacientes. Porém, ainda serão necessários novos estudos, já que existe bastante controvérsia a respeito de como integrar à prática clínica a informação obtida pelos estudos.

*Correspondência

Rua Joaquim Floriano, 72/24

São Paulo - SP

CEP: 04534-000

Tel: (11) 3168-7088 - Fax: (11) 3167-1148

\section{Referências}

1. OShaughnessy J, Twelves C, Aapro M. Treatment for anthracycline-pretreated metastatic breast cancer. Oncologist. 2002;7(Suppl 6):4-12.

2. Thomas ES, Gomez HL, Li RK, Chung HC, Fein LE, Chan VF, et al. Ixabepilone plus capecitabine for metastatic breast cancer progressing after anthracycline and taxane treatment. J Clin Oncol. 2007;25:5210-7.

3. Bonneterre J, Buzdar A, Nabholtz JM, Robertson JF, Thurlimann B, von Euler M, Sahmoud, et al. Anastrozole is superior to tamoxifen as first-line therapy in hormone receptor positive advanced breast carcinoma. Cancer. 2001;92:2247-58

4. Mouridsen $\mathrm{H}$, Gershanovich M, Sun Y, Perez-Carrion R, Boni C, Monnier A, et al. Phase III study of letrozole versus tamoxifen as first-line therapy of advanced breast cancer in postmenopausal women: analysis of survival and update of efficacy from the International Letrozole Breast Cancer Group. J Clin Oncol. 2003;21:2101-9.

5. Sargent DJ, Hayes DF. Assessing the measure of a new drug: is survival the only thing that matters? J Clin Oncol. 2008;26:1922-3.

6. Saad ED, Katz A, Buyse M. Overall survival and post-progression survival in advanced breast cancer: a review of recent randomized clinical trials. J Clin Oncol. 2010;28:1958-62.
7. Kaplan EM, P. Nonparametric estimation from incomplete observations. J Am Stat Assoc. 1958;53:457-81.

8. Smith I. Goals of treatment for patients with metastatic breast cancer. Semin Oncol. 2006;33:S2-5.

9. Sargent D. General and statistical hierarchy of appropriate biologic endpoints Oncology. 2006;20:5-9.

10. Hackshaw A, Knight A, Barrett-Lee P, Leonard R. Surrogate markers and survival in women receiving first-line combination anthracycline chemotherapy for advanced breast cancer. Br J Cancer. 2005;93:1215-21.

11. Di Leo A, Bleiberg $H$, Buyse $M$. Overall survival is not a realistic end point for clinical trials of new drugs in advanced solid tumors: a critical assessment based on recently reported phase III trials in colorectal and breast cancer. J Clin Oncol. 2003;21:2045-7.

12. Louvet C, Gramont A, Tournigand C, Artru P, Maindrault-Goebel F, Krulik M Correlation between progression free survival and response rate in patients with metastatic colorectal carcinoma. Cancer. 2001;91:2033-8.

13. Green S BJ, Crowley J. Clinical trials in oncology. London: Chapman \& Hall, 1997.

14. Saad ED, Katz A. Progression-free survival and time to progression as primary end points in advanced breast cancer: often used, sometimes loosely defined. Ann Oncol. 2009;20:460-4.

15. Saad ED, Katz A, Hoff PM, Buyse M. Progression-free survival as surrogate and as true end point: insights from the breast and colorectal cancer literature. Ann Oncol. 2010;21:7-12.

16. Miksad RA, Zietemann V, Gothe R, Schwarzer R, Conrads-Frank A, SchnellInderst $P$, et al. Progression-free survival as a surrogate endpoint in advanced breast cancer. Int J Technol Assess Health Care. 2008;24:371-83.

17. Burzykowski T, Buyse M, Piccart-Gebhart MJ, Sledge G, Carmichael J, Luck HJ, et al. Evaluation of tumor response, disease control, progression-free survival, and time to progression as potential surrogate end points in metastatic breast cancer. J Clin Oncol. 2008;26:1987-92.

18. Sherrill B, Amonkar M, Wu Y, Hirst C, Stein S, Walker M, et al Relationship between effects on time-to-disease progression and overall survival in studies of metastatic breast cancer. Br J Cancer. 2008;99:1572-8. 